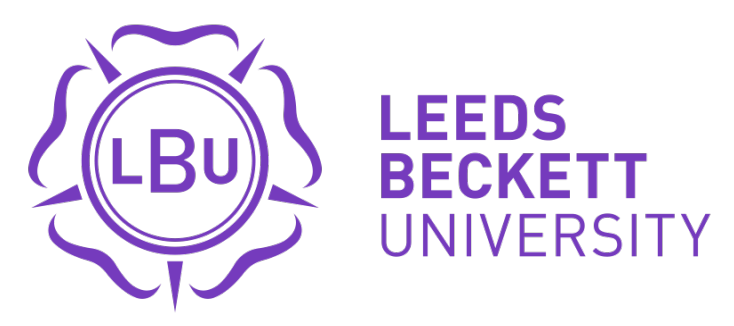

Citation:

Piggott, D and Boocock, E and Till, K and Abraham, A and Lara-Bercial, S (2016) International Sport Coaching Journal Digest. International Sport Coaching Journal / ISCJ, 3 (2). pp. 215-218. ISSN 2328-918X DOI: https://doi.org/10.1123/iscj.2016-0047

Link to Leeds Beckett Repository record:

https://eprints.leedsbeckett.ac.uk/id/eprint/3038/

Document Version:

Article (Accepted Version)

The aim of the Leeds Beckett Repository is to provide open access to our research, as required by funder policies and permitted by publishers and copyright law.

The Leeds Beckett repository holds a wide range of publications, each of which has been checked for copyright and the relevant embargo period has been applied by the Research Services team.

We operate on a standard take-down policy. If you are the author or publisher of an output and you would like it removed from the repository, please contact us and we will investigate on a case-by-case basis.

Each thesis in the repository has been cleared where necessary by the author for third party copyright. If you would like a thesis to be removed from the repository or believe there is an issue with copyright, please contact us on openaccess@leedsbeckett.ac.uk and we will investigate on a case-by-case basis. 


\section{INTERNATIONAL SPORT COACHING JOURNAL DIGEST VOLUME 3, ISSUE \#2}

Compiled by Dave Piggott, Emma Boocock, Kevin Till, Lea Dohme, Andrew Abraham and Sergio Lara-Bercial from the Sport Coaching and Physical Education Research Centre at Leeds Beckett University, UK

Digest contains a listing of pertinent, recent coaching and coach education articles and updates from other sources.

\section{Expert Coaches Perceptions of Athlete Performance Optimization}

Tracey, J. \& Elcombe, T. (2015). International Journal of Sports Science \& Coaching, 10(6), 1001-1014.

The purpose of the study was to engage expert coaches in a reflective process exploring their perceptions of distinguishing features of athletes they consider optimal performers. Fifteen national and/or Olympic level coaches were interviewed giving rise to a preliminary profile of elite athlete performance optimization centred on five main themes: a rational approach to training and performance; exhibiting sustained passion and commitment to details; ownership of the elite athletic experience; embracing challenge and complexity of elite athletic contexts; and employing a big (moral) picture view of sport and life. Discussion focuses on highlighting the features of performance optimizing athletes using the proposed concept of 'mature competitiveness', a quality that allows certain athletes to intentionally address the complex and highly contextual demands necessary to realistically optimize performance at the highest levels against peers of comparable athletic quality. The implications for coaches are also elaborated upon.

\section{Job and Life Satisfaction: The Perspective of Collegiate Head Coaches}

Bopp, T., Wigley, B.J., \& Eddosary, M. (2015). International Journal of Sports Science \& Coaching, 10(6), 1025-1038.

The purpose of this research was to further expand on the relationship between job and life satisfaction among National Collegiate Athletic Association (NCAA) head coaches. Data from 348 head coaches across all three NCAA Divisions were analyzed. Results imply that life satisfaction and job satisfaction are significantly and positively related in the collegiate coaching context. However, the relationship between the two constructs was not reciprocal and the unidirectional effects differed based on the gender of the team coached. Practical implications of these findings might offer significant insights for managers and policy makers such that they are better equipped to establish and increase work (e.g., job) and non-work (e.g., life) satisfaction.

\section{Elite Coach Perceptions of Cohesion on Coacting Teams}


Cormier, M.L., Bloom, G.A. and Harvey, W.J. (2015). International Journal of Sports Science \& Coaching, 10(6), 1039-1054.

This study explored the knowledge and perceptions of elite coacting sport coaches as applied to team cohesion. Six experienced university crosscountry running coaches who achieved high levels of success were interviewed for this study. Results demonstrated that these coaches valued cohesion and felt it played an integral role in developing team success and satisfaction, despite facing barriers such as intrateam competition and rivalry. Purposeful recruiting, choosing effective team leaders, setting and monitoring team goals, and encouraging social events were some strategies utilized to enhance cohesion and establish positive relationships among teammates. These findings provide a greater understanding of the knowledge of elite coacting coaches and cohesion and how coaches can enhance it in their teams.

\section{Knowledge Translation of Sport Psychology to Coaches: Coaches' Use of Online Resources}

Pope, J.P., Westlund Stewart, N., Law, B., Hall, C.R., Gregg, M.J. \& Robertson, R. International Journal of Sports Science \& Coaching, 10(6), 1055-1070.

The purpose of this study was to examine the content of the information coaches attain from online sport psychology resources and their use of this information. This authors investigated differences in coaches' use of online resources across experience, certification, and competitive level of the coaches. Results demonstrated that coaches currently get information from online sport psychology resources "a few times per year", but would get it "once per month" if more accessible and credible resources were available. The study findings also indicated that coaches would be interested in accessing applied information such as sport psychology tips, skills/strategies, and how to implement sport psychology with their athletes.

\section{Perceived Teammate Acceptance and Sport Commitment in Adolescent Female Volleyball Players}

Garn, J. (2016). The Sport Psychologist, 30(1), 30-39.

Grounded in Scanlan's sport commitment model (SCM), this study explored the relationship between feelings of teammate acceptance and sport commitment in a sample of adolescent female volleyball players. Direct and indirect relationships between teammate acceptance and sport commitment within the SCM were tested. Findings supported the indirect relationship between teammate acceptance and sport commitment through sport enjoyment, personal investments, social constraints, and investment opportunities, accounting for $48 \%$ of the variance in sport commitment. It therefore appears that teammate acceptance may be better situated as a distal 
source of sport commitment, but the authors stressed that further research with more diverse samples is necessary. The development of responsive interpersonal skills in athletes, coaches, and parents, and the reduction of corporal punishment and aggression tactics is proposed to facilitate greater levels of social acceptance.

\section{Enhancing self-efficacy through a blended training: A pilot study with basketball players.}

Villani, D., Caputo, M., Balzarottu, S., \& Riva, G. (2015). International Journal of Sport and Exercise Psychology, 1, 1-16.

This study aimed to test the effectiveness of a blended training program that combined face-toface and web-based activities in enhancing self-efficacy and emotion regulation in young basketball players. A two-group pre- and post-test design was used, wherein 37 basketball players received either a web-based or a control text-based training program. The program lasted seven weeks. Results indicated that athletes receiving the web-based training reported higher levels of self-efficacy, while no significant difference was found in the control group. Concerning emotional regulation, both groups reported less emotional suppression at the end of the program. In relation to the practical implementation within youth coaching, this indicates that web-based technology can be effectively employed to support training aimed at enhancing psychological skills such as self-efficacy.

\section{Athletes' perception of coaching behaviour, relation-inferred self-efficacy (RISE), and self-efficacy in youth sport.}

Saville, P.D. \& Bray, S.R. (2016). Journal of Applied Sport Psychology, 28, 1-13.

This quantitative study aimed to investigate the associations between coaching behaviours, relation-inferred self-efficacy (RISE: A person's perception about what another person believes about his/her capabilities), and self-efficacy in youth sport. In total, 277 youth athletes completed questionnaires measuring coaching behaviours, self-efficacy, and RISE. Results indicated that RISE-relevant coaching behaviours (e.g., reinforcing hard-work over winner, praising effort, etc.) showed positive associations with athletes' RISE and self-efficacy beliefs. In relation to the practical implementation within youth coaching, these findings support the important role of coaching behaviours and RISE perceptions as a pathway to predicting self-efficacy in youth athletes. This helps to further understand the ways in which youth athletes develop beliefs of their personal sport abilities.

\section{An exploration of the academic coaching education internship.}

Zakrajsek, R., Thompson, M. \& Dieffenbach, K. (2015). Sports Coaching Review, 4(1), 24-40. 
Within this article the authors investigate the academic programmes in the USA that prepare the student coaches for the coaching profession. The research here aims to address the lack of knowledge which is known about coach education programmes, how they are delivered and their effectiveness when preparing coaches for the realities of the idiosyncratic nature of coaching. 137 coach education programme were identified. However, only 59 responded to the call for participation. The authors collected survey data from all 59 participants which was made up of five sections, each relating to programme development (Academic Athletic Coaching Education Programme Coordinator; Coaching Education Curriculum; Internship/Practicum Experience; Student Coach Internship Opportunities; Mentor Coach). The study found that there were several causes for concern within the coach education programmes within the USA, especially within the internship programme. The authors highlighted that there was a lack of consistency in the delivery of the programmes; the programme had higher expectations of their student-coaches' experiences and that there was a limited evaluation of the mentor coaches. All in all, this article serves to support the need for more evaluation work to be conducted in coach education to address the need for developing coach education programmes for future coaches.

\section{In pursuit of becoming a senior coach: the learning culture for Australian Football League coaches}

Mallett, C.J., Rossib, T., Rynne, S.B. \& Tinning, R. (2015). Physical Education and Sport Pedagogy 21(1), 24-39.

Understanding the learning and development of senior coaches (SCs) and assistant coaches (ACs) in the Australian Football League is important to better develop the next generation of performance coaches. Hence the focus of this research was to examine the learning of SC and AC in the AFL. Fundamental to this research was an understanding that the AFL and each club within the league be regarded as learning organisations and workplaces with their own learning cultures where learning takes place. The purpose of this paper was to examine the learning culture for AFL coaches. The authors found that although learning was central to AFL coaches becoming a SC, coaches reported a sense of isolation and a lack of support in developing their craft within their particular learning culture. These coaches developed a unique dynamic social network (DSN) that involved episodic contact with a number of respected confidantes often from diverse fields in developing their coaching craft. Although there were some opportunities in their workplace, much

of their learning was unmediated by others, underscoring the importance of their agentic engagement in limited workplace affordances.

\section{Valued learning experiences of early career and experienced high-performance coaches}

Mallett, C.J., Rynne, S.B. \& Billett, S. (2016). Physical Education and Sport Pedagogy, 21(1), 89104. 
This paper attempts to move the discussion of high performance coach development from an examination of coaches' volume of experiences towards a consideration of the contribution of the learning experiences that coaches have reported throughout their careers. Furthermore, a discussion of proximal and distal guidance in the development of coaches was investigated. The authors examined the kinds of learning experiences within the framework of workplace learning and specifically the situated nature of learning and the view that learning occurs through social participation. Participants (mentor coaches and scholarship coaches) rated a list of 14 developmental activities derived from empirical research in the first two years of their coaching career, middle two years, and final two years. Three key findings emerged from these data: (i) reported increased valuing of a range of developmental experiences over time; (ii) temporal variance in the value of different learning sources at different stages of their careers; and (iii) an acknowledgement of the shift away from an emphasis on proximal learning sources for the mentor coaches and the shift towards proximal sources for the scholarship coach.

\section{A coach's political use of video-based feedback: a case study in elite-level academy soccer.} Booroff, M., Nelson, L. \& Potrac, P. (2016). Journal of Sports Sciences, 34, 116-124.

This paper explores a single coach's everyday use of performance analysis in a specific organisational context (elite U18s academy soccer in the UK). The coach in question was studied over a whole season, with data collection comprising an initial 4 interviews followed by 10 videofeedback session observations, before 2 final interviews. The data revealed that the coach used video reviews for three main strategic reasons: 1) to provide augmented feedback targeted specifically at developing the best players (those most likely to be sold or to play in the first team); 2) to create a more professional and 'serious' atmosphere to prepare young players for the highly competitive adult game; and 3) to provide hard evidence to support the release of players. The authors conclude by inviting readers to consider the highly politicised ways in which coaches engage with performance analysis to achieve far broader strategic objectives than the research in this field has previously considered.

\section{The microstructure of coaching practice: behaviours and activities of an elite rugby union head coach during preparation and competition.}

Hall, E. T., Gray, D. \& Sproule, J. (2016). Journal of Sports Sciences, 34, 896-905.

This study took a descriptive exploratory approach to examining the practice of a female head coach of a national rugby union team. The coach was recorded in 14 training sessions and 6 games over the course of a season. The authors analysed practice using a systematic observation instrument adapted to rugby, which involved the coding of both coaching behaviours and practice forms. The results showed a high degree of inter-session variability in practice forms, but, contrary 
to previous research, with slightly more playing form (58.5\%) compared to training form (41.5\%) activity on average. As would be expected, the coached used far more pedagogical behaviours (e.g. instructing and questioning) during practice compared to matches, where they were more likely to 'observe' and 'confer with associates'. In conclusion, the authors suggest that the coach used a more facilitatory and playing form approach than has been found in previous studies. They also argue that researchers need to study coaches' interactions with colleagues and the subsequent impact on practice decisions as this is a poorly understood yet growing part of an elite coach's role.

\section{Effect of Small-Sided Games and Repeated Shuffle Sprint Training on Physical Performance in Elite Handball Players.}

Dello Iacono, A., Ardigo, L.P., Meckel, Y., \& Padulo, J. (2016) J Strength Cond Res 30(3), 830840.

To develop the physical performance of team sport athletes a range of training methodologies are available. In elite handball, two training methods include small sided games (SSGs) and repeated shuffle sprint (RSS) training but the most effective training method is currently unknown. Therefore, this study compared the effects of SSGs and RSS training on sprint speed, agility, repeated sprint ability, countermovement jump, standing throw and jump shot speed in adult elite handball players. Eighteen highly trained players $(24.8 \pm 4.4$ years $)$ were assigned to either SSG or RSS group training protocols twice a week for 8 weeks. The SSG training consisted of 5 smallsided handball games with 3-a-side teams excluding goalkeepers. The RSS consisted of 2 sets of 14-17 of 20-m shuttle sprints and 9-m jump shots interspersed by 20 -second recoveries. Both training groups significantly improved all physical performance characteristics across the 8-week programme. RSS training significantly improved 10m sprint, countermovement jump and jump shooting compared to SSGs. SSGs demonstrated significantly greater improvements in agility and standing throw performance than RSS training. These findings suggest that both SSGs and RSS training are effective training methods for developing physical performance in handball. Coaches should consider the use of both training methods for maximising physical performance within handball. 